\title{
Star-formation in nuclear clusters and the origin of the Galactic center apparent core distribution
}

\author{
Danor Aharon and Hagai B. Perets \\ Physics Department, Technion - Israel Institute of Technology, Haifa 3200003, Israel \\ email: danor@tx.technion.ac.il
}

\begin{abstract}
Nuclear stellar clusters (NSCs) are known to exist around massive black holes (MBHs) in galactic nuclei. Two formation scenarios were suggested for their origin: build-up of NSCs and Continuous in-situ star-formation. Here we study the effects of star formation on the build-up of NSCs and its implications for their long term evolution and their resulting structure. We show that continuous star-formation can lead to the build-up of an NSC with properties similar to those of the Milky-way NSC. We also find that the general structure of the old stellar population in the NSC with in-situ star-formation could be very similar to the steady-state Bahcall-Wolf cuspy structure. However, its younger stellar population does not yet achieve a steady state. In particular, formed/evolved NSCs with in-situ star-formation contain differential age-segregated stellar populations which are not yet fully mixed. Younger stellar populations formed in the outer regions of the NSC have a cuspy structure towards the NSC outskirts, while showing a core-like distribution inwards; with younger populations having larger core sizes.
\end{abstract}

\section{Introduction}

Nuclear stellar clusters (NSCs), hosting massive black holes (MBHs) are thought to exist in a significant fraction of all galactic nuclei. Their origin is still not well understood. Two main scenarios were suggested for their origin: (1) The cluster infall scenario, in which stellar clusters inspiral to the galactic nucleus are disrupted, and thereby build up the nuclear cluster (Tremaine et al. (1975); Antonini (2013)). (2) The nuclear star formation (SF) scenario, in which gas infalls into the nucleus and then transforms into stars through star formation processes (Loose et al. (1982)). Here we focus on the latter process, and study the long term effects of SF on the formation and evolution of NSCs.

The structure, evolution and dynamics of NSCs have been extensively studied in recent years. These studies explored the general dynamics of NSCs, and in particular NSCs similar to the well-observed NSC in the Milky Way Galactic Center (GC). The presence of a young stellar disk in the central pc of GC, as well as a dense concentration of HII regions and young stars throughout the central $100 \mathrm{pc}$ of the Milky-way (Figer et al. (2004)) provide evidence for a continuous star-formation in this region (Genzel et al. (2010)). Evidence for star formation exists in other extagalactic NSCs (e.g. Seth et al. (2006)). Walcher et al. (2005) argued that NSCs are protobulges that grow by repeated accretion of gas and subsequent star formation, McLaughlin et al. (2006) suggested a NSC in-situ star formation model regulated by momentum feedback.

These various studies provide further motivation and suggest that star-formation has an important role in shaping NSCs and their evolution. Here, we summarize our results of the role of in-situ SF in NSCs, and explore its implication both for the build-up of the NSC, as well as the long term evolution and structure of NSCs (detailed information can 
be found in Aharon \& Perets (2014)). Our work makes use of the Fokker-Planck (FP) diffusion equation, first used by Bahcall \& Wolf (1976) in this context, to describe the dynamics of stellar populations in dense clusters around MBHs.

\section{Evolution of NSCs around MBHs: by Fokker-Planck analysis}

NSCs are complex interacting systems. Their evolution and dynamics are mainly affected by two-body relaxation. Here we follow the evolution of NSCs by numerically solving the FP equation following the approach first used by Bahcall \& Wolf (BW; 1976, 1977) in this context. However, we supplement the basic equation, for the first time, with a source term accounting for $\mathrm{SF}$, as well as use a large number of distinct stellar populations to account for different SF epochs.

In our model we simulate the star formation in the GC through adding an extra source term component to the $\mathrm{FP}$ equation. Its value and range are determined according to the number of new stars added in the appropriate region. We simulate multiple stellar populations forming at different epochs, and follow the evolution of their distribution. The modified FP equation with the addition of the source term has the form:

$$
\frac{\partial f(E, t)}{\partial t}=-A E^{-\frac{5}{2}} \frac{\partial F}{\partial E}-F_{L C}(E, t)+F_{S F}
$$

where the $F_{L C}(E, t)$ term corresponds to the empty loss-cone term (see Lightman \& Shapiro (1977); Young (1977); Perets et al. (2007)).

The source term added to the FP equation is:

$$
F_{S F}=\frac{\partial}{\partial t}\left(\Pi(E) E_{0} E^{\alpha}\right)
$$

where $\Pi(E)$ is a rectangular function, which boundaries correspond to the region where new stars are assumed to from; $E_{0}$ is the source term amplitude; and $F_{S F}$ is a powerlaw function with a slope $\alpha$, defining the SF distribution in phase space. We simulated a number of NSC evolutionary scenarios, taking different models for the SF function (rate and spatial structure) and for the background population. The chosen slope of the SF function was motivated by the observed power-law (Do et al. (2009)) distribution of young stars observed in the young stellar disk in the GC.

\section{Results}

We have followed the evolution of multiple stellar populations formed at different epochs. The build-up and structure evolution of an NSC which grows through a continuous long-term in-situ star formation. The final configuration of this NSC is very similar to that of a steady state BW cusp, and the number densities are comparable to those observed in the GC. The final structures of these NSCs after 10 Gyr of evolution for the two main scenarios ( $2 \mathrm{~b}$ and 7 ) are summarized in Fig. 1 . The other scenarios can be found in Aharon \& Perets (2014). These models show the existence of a core-like structure for the young stellar populations, where the cores vary in size, and are systematically bigger for younger populations.

\section{Discussion}

The build up of nuclear stellar clusters through in-situ star formation. We explored two evolutionary scenarios of NSCs: (1) pre-existing NSCs with a BW-like structure that experience later SF and (2) NSCs built-up completely from in-situ SF. Both type of models 

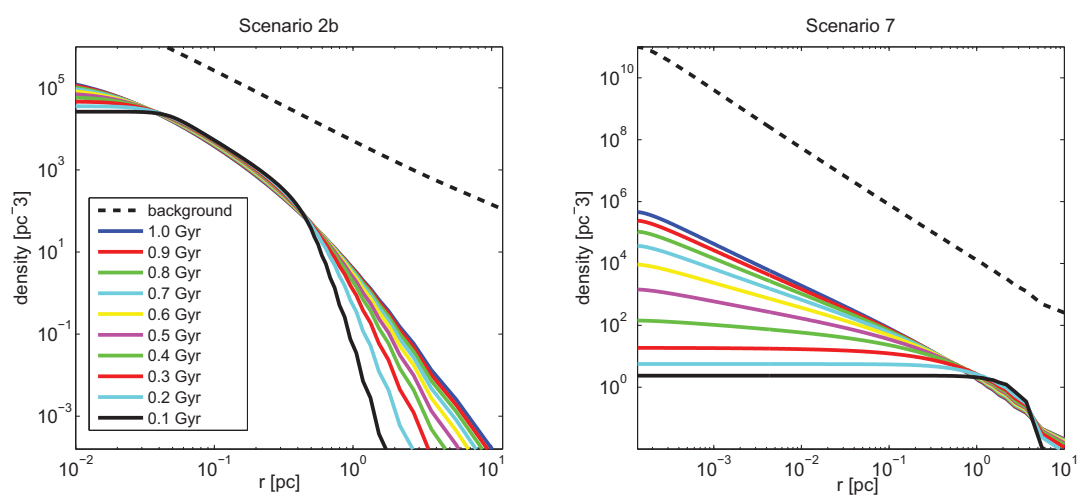

Figure 1. The number density profile of a 10 Gyr evolved NSC with SF inside the central $2 \mathrm{pc}$ (scenario $2 \mathrm{~b}$ ) and outside (Scenario 7 ). The number of gigayears presents the age of the new population. The difference in the SF range affects the final distribution of an evolved NSC. The old background stellar population (black dashed lines) correspond either to stars produced through in-situ SF (over the first 9 Gyr; right) or to a pre-existing BW cusp population (left). The structures of these old populations show an almost BW-like steady state behavior in both of the models, while the young stellar populations formed in the last Gyr are not yet relaxed, and show large cores ranging in size. The young populations arise from in-situ star formation

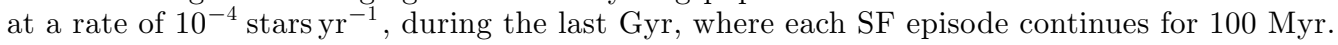

assume that several epochs of gas-infall into the nuclear region triggered SF, transforming the infalling gas into newly born stars. We show that NSCs built-up from in-situ SF give rise to NSCs dominated by the stellar population formed at earlier stages (first few gigayears). The structure of the older population is very similar to a steady-state BW-cusp, and the total number of stars is comparable to that inferred for the GC NSC. We note that when lower mass stellar populations are assumed (e.g. if different initial mass function are considered) the relaxation times become longer, as expected, and lateformed younger populations are far from achieving a steady state structure, producing larger core-like structures, as discussed in more details below.

Core-cusp structure. After $\sim 10 \mathrm{Gyr}$ of NSC evolution, the younger stellar populations in NSCs may evolve to a core-like distribution, while older population already become progressively cuspier. These results are of great interest in light of the recent findings about the structure of the GC NSC.

There are a number of models suggesting to explain the origin of the GC core, for example Merritt (2010) suggested that a binary merger, or a triaxial potential could deplete the inner regions of an NSC producing a large core, and have shown that the long relaxation times would not be sufficient to regrow a cusp. A similar behavior is seen in our models, where progressively younger populations of stars formed in the outer regions of the NSC do not relax and grow an inner cusp. Though in both cases slow relaxation explains the non-growth of the inner cusp, the origins of the initial core in both models differ, and the outcomes could significantly differ as well. In particular, the SF models studied here suggest that cores of different sizes could exist for different stellar populations, and in particular an NSC can have both a cusp distribution of old stars and a core distribution for young and intermediate age stars.

We note that younger, more massive red giants could be more luminous and more easily detected in observations (Pfuhl et al. (2011)). We therefore hypothesize that if such younger red giants (up to 2-3 Gyr old) are overly represented in observations then the observed core could be limited to these younger populations, while the underlying population of older stars might still have a cusp distribution. This can be well 


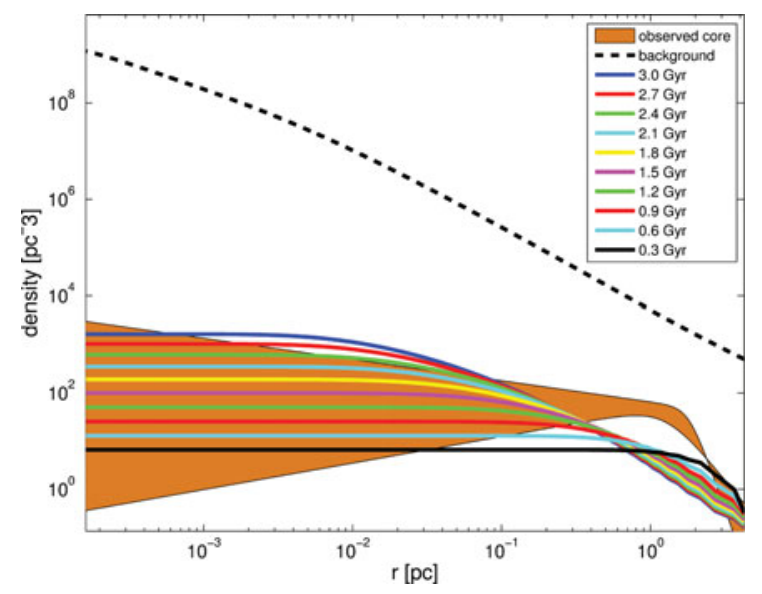

Figure 2. The number density profile of the GC nuclear cluster after 10 Gyr evolution (and total star formation epoch of $3 \mathrm{Gyr}$ ) compared to the inferred range of number density profiles from 3D modeling of GC observations Do et al. (2013). The number of Gyrs presents the age of the new population.

demonstrated both in Fig. 2 where the model results are compared with the 3D modeled number density profile determined by Do et al. (2013) based on observations. As can be seen, in some models a large, parsec-size core of up to a few gigayears old stellar populations can exist. Such a core might be consistent with the density profiles inferred from observations, while the old stellar population preserves a typical BW-like cusp profile.

\section{References}

Aharon, D. \& Perets, H.B. 2014 ArXiv e-prints, 1409.5121

Antonini, F. 2013, ApJ, 763, 62

Bahcall, J. N. \& Wolf, R. A. 1976, ApJ, 209, 214-232

Bahcall, J. N. \& Wolf, R. A. 1977, ApJ, 216, 883-907

Do, T., Ghez, A. M., Morris, M. R., Lu, J. R., Matthews, K., Yelda, S., \& Larkin, J. 2009, ApJ, 703, 1323-1337

Do, T., Martinez, G. D., Yelda, S., Ghez, A., Bullock, J., Kaplinghat, M., Lu, J. R., Peter, A. H. G.., \& Phifer, K. 2013, ApJL, 779, L6

Figer, D. F., Rich, R. M., Kim, S. S., Morris, M., \& Serabyn, E. 2004, ApJ, 601, 319-339

Genzel, R., Eisenhauer, F., \& Gillessen, S. 2010, Reviews of Modern Physics, 82, 3121-3195

Lightman, A.P. \& Shapiro, S.L. 1977 ApJ, 211, 244-262

Loose, H. H., Kruegel, E., \& Tutukov, A. 1982, AAP, 105, 342-350

McLaughlin, D. E., King, A. R., \& Nayakshin, S. 2006, ApJL, 650, L37-L40

Merritt, D 2010, ApJ, 718, 739-761

Perets, H. B., Hopman, C., \& Alexander, T. 2007, ApJ, 656, 709-720

Pfuhl, O., Fritz, T. K., Zilka, M., Maness, H., Eisenhauer, F., Genzel, R., Gillessen, S., Ott, T., Dodds-Eden, K., \& Sternberg, A. 2011, ApJ, 741, 108

Seth, A. C., Dalcanton, J. J., Hodge, P. W., \& Debattista, V. P. 2006, AJ, 132, 2539-2555

Tremaine, S.D., Ostriker, J.P., \& Spitzer, L. Jr. 1975 ApJ, 196, 407-411

Walcher, C. J., Van der Marel, R. P., McLaughlin, D., Rix, H. W., Böker, T., Häring, N., Ho, L. C., Sarzi, M., \& Shields, J. C. 2005, ApJ, 618, 237-246

Young, P. J. 1977, ApJ, 215, 36-52 